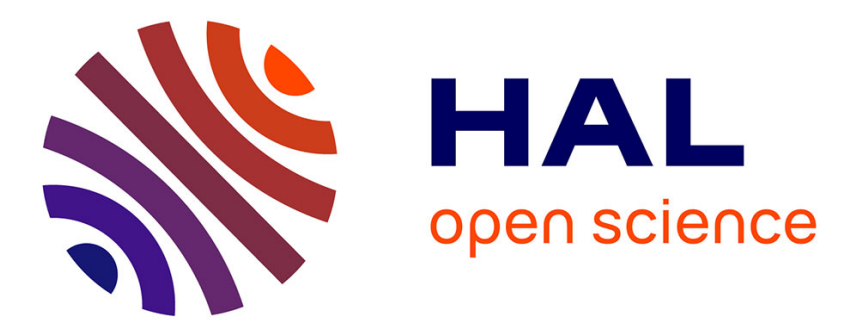

\title{
Unsteady dynamics of PAH and soot particles in laminar counterflow diffusion flames
}

Pedro Rodrigues, Benedetta Franzelli, Ronan Vicquelin, Olivier Gicquel, Nasser Darabiha

\section{- To cite this version:}

Pedro Rodrigues, Benedetta Franzelli, Ronan Vicquelin, Olivier Gicquel, Nasser Darabiha. Unsteady dynamics of PAH and soot particles in laminar counterflow diffusion flames. Proceedings of the Combustion Institute, 2017, 36 (1), pp.927 - 934. 10.1016/j.proci.2016.07.047 . hal-01480263

\section{HAL Id: hal-01480263 https://hal.science/hal-01480263}

Submitted on 13 Mar 2017

HAL is a multi-disciplinary open access archive for the deposit and dissemination of scientific research documents, whether they are published or not. The documents may come from teaching and research institutions in France or abroad, or from public or private research centers.
L'archive ouverte pluridisciplinaire HAL, est destinée au dépôt et à la diffusion de documents scientifiques de niveau recherche, publiés ou non, émanant des établissements d'enseignement et de recherche français ou étrangers, des laboratoires publics ou privés. 


\title{
Unsteady dynamics of PAH and soot particles in laminar counterflow diffusion flames
}

\author{
Pedro RODRIGUES ${ }^{\mathrm{a}, *}$, Benedetta FRANZELLI ${ }^{\mathrm{a}}$, Ronan VICQUELIN ${ }^{\mathrm{a}}$, Olivier GICQUEL ${ }^{\mathrm{a}}$, Nasser DARABIHA \\ ${ }^{a}$ Laboratoire EM2C, CNRS, CentraleSupélec, Université Paris-Saclay, \\ Grande Voie des Vignes \\ 92295 Châtenay-Malabry Cedex, France
}

\begin{abstract}
Due to their low chemical time scales, the production of soot particles in turbulent diffusion flames is highly impacted by large range of local strain rate fluctuations.

In order to understand the response of soot production to strain rate fluctuations, unsteady laminar counterflow diffusion flames with an imposed oscillating strain rate are investigated both analytically and numerically. First an analytical linearized model is developed to predict the unsteady response of a flame quantity of interest from information on laminar steady flames. Three critical parameters governing flame response are identified: the Stokes number which compares the characteristic time associated to the mean imposed strain rate to the oscillation frequency, the Damköhler number associated to the quantity of interest, and a third one characterizing the response of this quantity to an imposed steady strain rate. This model is then applied to soot predictions. Parallely, the response of soot production in propane-air counterflow diffusion flames to unsteady strain harmonic oscillations is studied numerically using a detailed sectional soot model. A wide range of frequencies and amplitudes are considered. A specific trend is highlighted for soot precursors and particles production according to their respective chemical time scales: the bigger the PAH or soot particle, the higher its chemical time scale, resulting in a more damped and phase-lagged response. The particle size distribution evolves accordingly during the considered oscillations, so that the quasi-steady state behaviour is not verified for high frequencies. The numerical results are compared to those obtained by the analytical approach and a very good agreement is obtained at low amplitudes. Non-linear response of soot precursors and soot particles production to strain oscillations are finally discussed in case of high oscillation amplitudes and the limits of the proposed analytical model are identified.
\end{abstract}

Keywords: Soot, PAH, Laminar flame dynamics, Soot sectional model, Particle size distribution

\section{Introduction}

Due to incomplete combustion, soot emissions have effects on both human health and environment. Soot emissions are also considered as an important cause of global warming [1]. Consequently, important efforts are made both experimentally and numerically [2, 3, 4] to understand soot production mechanisms in order to control their emission.

Most of the combustion facilities are characterized by high Reynolds number flames where turbulent eddies are expected. The local strain rate usually fluctuates in a

${ }^{*}$ Corresponding author:

Email address: pedro.rodrigues@centraliens.net (Pedro RODRIGUES)

Preprint submitted to Proceedings of the Combustion Institute wide amplitude range and with random fluctuation frequencies [5]. These turbulent eddies are also responsible for variable length scale recirculation zones, introducing a wide range of residence times for soot particles, strong intermittency and dynamics features in soot production $[6,7]$.

One of the most popular approaches used to simulate turbulent non-premixed flames is the flamelet approach, based on a quasi-steady response of the flame characteristics to the local strain rate fluctuations $[8,9]$.

In the optic of applying such models to numerical simulations of turbulent flames, the response of soot to strain rate fluctuations can be investigated by looking at unsteady laminar counterflow diffusion flames $[10,11,12]$. Specifically to soot context, previous experimental works have been performed in a diffusion

March 13, 2017 
laminar flame by introducing sinusoidal velocity variations at both opposed nozzles [13,14] . They showed that soot production response to these fluctuations was phase-lagged and damped when increasing the oscillation frequency. A particular hierarchical behavior was observed: soot volume fraction response is more phaselagged and damped compared to soot precursors response, which are also more phase-lagged and damped than the temperature response [15]. Cuoci et al. [16] numerically investigated these flames with good prediction of unsteadiness soot dynamics, confirming the experimental observations. Nevertheless, a lack of knowledge remained on the origin of soot response to unsteady strain fluctuations. Moreover, when computing counterflow diffusion flames with unsteady velocities at the nozzle exits, a phase lag exists between the global strain rate and the local strain rate [16], increasing the complexity of the phenomena.

The objective of the present work is to characterize the response of soot to strain rate oscillations and to identify the physical phenomena underlying the phase lag and damping observed in soot production. In order to avoid the phase lag between the global and the local strain rate, a strain-imposed formulation is considered in this work and unsteadiness is introduced by varying the imposed flame strain rate $a(t)$ with time for a given pulsation $\omega$, an initial strain rate $A_{0}$ and fluctuation amplitude $a_{1}$ :

$$
a(t)=A_{0}+a_{1} \sin (\omega t)=A_{0}[1+\alpha \sin (2 \pi f t)] .
$$

Both analytical and numerical approaches are considered in this paper to study the evolution of the soot precursors and of the particle size distribution (PSD) with the strain rate $a(t)$.

The paper is organized as follows. First, an analytical model is proposed in Section 2 in the limit of a linear behavior, i.e. small oscillation amplitudes. This model predicts the unsteady response on the basis of steady flame results. Then, soot production in unsteady laminar flames is numerically studied using a detailed sectional model. The modeling strategy is introduced in Section 3. The flame response is then investigated for the configuration described in Section 4.1. The unsteady behavior is analyzed in Section 4.2 for different frequencies at small amplitude in terms of global quantities and PSD. Analytical results will be compared to the numerical ones in Section 4.3 to prove their validity. The causes of phase lag and damping in soot production will then be identified by combining information from numerical and analytical results. Finally, numerical simulations at high amplitudes are analyzed in Section 4.4 to completely characterize the soot response to unsteady strain rate oscillations and to discuss the limits of the analytical model.

\section{Analytical model for pulsed sooted flames}

In order to investigate the response of soot production to strain rate fluctuations, a linearized analytical model is developed in the following to predict the response of the maximum of a flame variable $\theta$ to strain rate oscillations at a given pulsation $\omega$. The complex form of the fluctuating strain rate $a_{1}(t)=a(t)-A_{0}$ is denoted by $\hat{a}_{1}(\omega)=\alpha A_{0} e^{i(\omega t+\pi / 2)}$. The corresponding response of the maximum value of $\theta$, namely $\theta^{\max }(t)=\theta_{0}^{\max }+\theta_{1}^{\max }(t)$ with $\theta_{1}^{\max }(t)=$ $\theta_{1}^{\max }(\omega) \sin \left(\omega t-\varphi_{\theta^{\max }}(\omega)\right)$, is represented by the complex number $\hat{\theta}_{1}^{\max }(\omega)=\theta_{1}^{\max }(\omega) e^{i\left(\omega t+\pi / 2-\varphi_{\theta} \max (\omega)\right)}$. This response is fully characterized by the transfer function $T_{\theta^{\max }}(\omega)=\hat{\theta}_{1}^{\max }(\omega) / \hat{a}_{1}(\omega)$.

Starting from the previous works $[10,11,12]$, the transfer function is split into two terms: the transfer function $T_{\text {unst }}^{\text {finite } \theta}(\omega)$, introducing an equivalent steady strain rate $A_{\theta}$ seen by the quantity $\theta$, and the transfer function $T_{\text {steady }}^{\theta^{\max } \mid A_{\theta}}(\omega)$, describing the response of $\theta^{\max }$ to the equivalent steady strain rate $A_{\theta}$.

\subsection{Equivalent steady strain rate}

Following [10, 11, 12], under the assumption of infinitely fast chemistry, the unsteady flame acts at each time $t$ as an equivalent steady counterflow flame at constant strain rate equal to the instantaneous strain rate $A(t)$ verifying:

$$
\frac{d A}{d t}=-2 A^{2}(t)+2 A(t) a(t)
$$

Assuming a linear response of $A(t)=A_{0}+A_{1}(t)$ with $a(t)=A_{0}+a_{1}(t)$, i.e. small fluctuations of $a(t)$ around $A_{0}$, the transfer function $T_{\text {unst }}^{\text {inf }}(\omega)$ between $\hat{A}_{1}(\omega)$ and $\hat{a}_{1}(\omega)$ in the case of infinitely fast chemistry is given by:

$$
T_{\mathrm{unst}}^{\mathrm{inf}}(\omega)=\frac{1}{1+j \omega /\left(2 A_{0}\right)} .
$$

When finite-rate chemistry is considered, the equivalent strain rate $A_{\theta}$ for a given variable $\theta$ ( $T$ or $Y_{k}$ ) is given by [10]:

$$
\frac{\partial A_{\theta}}{\partial t}=\frac{A_{\theta}(t)-A(t)}{A_{\theta}(t)} \frac{\dot{\Omega}_{\theta}(t)}{(d \theta / d A)_{\Omega}}=\frac{A(t)-A_{\theta}(t)}{\gamma_{\theta}(t)},
$$

where $\dot{\Omega}_{\theta}=\dot{\omega}_{T}^{\max } /\left(\rho c_{p}\right)$ for $\theta=T$ and $\dot{\Omega}_{\theta}=W_{k} \dot{\omega}_{k}^{\max } / \rho$ for $\theta=Y_{k} . \rho$ and $c_{p}$ are evaluated at the position where $\dot{\omega}_{\theta}$ is maximum. $(d \theta / d A) \Omega$ represents the variation of $\theta$ 
where $\dot{\omega}_{\theta}$ is maximum with a steady strain rate. $\gamma_{\theta}(t)$ is defined as $\gamma_{\theta}(t)=-(d \theta / d A) \Omega^{A_{\theta}}(t) / \dot{\Omega}_{\theta}(t)$.

To find the linearized response of $A_{\theta}(t), A_{\theta}(t)$ and $\gamma_{\theta}(t)$ are written as: $A_{\theta}(t)=A_{0}+A_{\theta_{1}}(t)$ and $\gamma_{\theta}(t)=$ $\gamma_{\theta_{0}}+\gamma_{\theta_{1}}(t)$, where $A_{0}, \gamma_{\theta_{0}}$, are the values of respectively $A_{\theta}(t)$, and $\gamma_{\theta}(t)$ for the initial steady flame. By linearizing Eq. (4), one obtains:

$$
\frac{\partial A_{\theta_{1}}}{\partial t}=\left(A_{1}(t)-A_{\theta_{1}}(t)\right) \gamma_{\theta_{0}}^{-1}
$$

Combining the Fourier transform of Eq. (5) and Eq. (3), the following transfer function $T_{\text {unst }}^{\text {finite }, \theta}(\omega)$ between $\hat{A}_{\theta_{1}}(\omega)$ and $\hat{a}_{1}(\omega)$ is obtained:

$$
T_{\mathrm{unst}}^{\mathrm{finite}, \theta}(\omega)=\frac{\hat{A}_{\theta_{1}}(\omega)}{\hat{a}_{1}(\omega)}=\frac{1}{1+j \omega \gamma_{\theta_{0}}} \frac{1}{1+j \omega /\left(2 A_{0}\right)} .
$$

\subsection{Steady response of the maximum value}

Once the equivalent steady strain rate $A_{\theta}(t)$ is known, the flame response can be analyzed by looking at steady conditions. For $\theta \in\left\{T, Y_{k}\right\}$, it has been observed that in the neighborhood of a given strain rate $A_{0}$, the dependency of $\theta^{\max }$ for a steady flame with strain rate $A$ is given by [17]:

$$
\left(\theta^{\max }(A) / \theta^{\max }\left(A_{0}\right)\right)=\left(A / A_{0}\right)^{p_{\theta}}
$$

with $p_{\theta}$ a characteristic constant. Linearizing Eq. (7) with $\theta^{\max }(t)=\theta_{0}^{\max }+\theta_{1}^{\max }(t)$ gives $\theta_{1}^{\max }(t)=\frac{p_{\theta} \theta_{0}^{\max }}{A_{0}} A_{\theta}(t)$.

However, the forthcoming comparison with the detailed computation demonstrates the requirement to introduce an additional delay in the response to the unsteady strain rate oscillations. Linking this delay to the chemical time seems particularly relevant for soot precursors and particles, whose chemistry is mainly sequential so that all the reactions necessary for the formation have to respond before getting the response of $\theta^{\max }$. This delay is then assumed to be equal to the characteristic chemical time scale $\tau_{\theta}$ of the quantity of interest $\theta$ defined in Appendix A: $\theta^{\max }(t)$ reacts then at the equivalent strain rate $A_{\theta}\left(t-\tau_{\theta}\right)$. The validity of this hypothesis will be verified in Section 4.3. The response of $\theta_{1}^{\max }(t)$ is therefore expressed as:

$$
\begin{aligned}
& \theta_{1}^{\max }(t)=p_{\theta} \theta_{0}^{\max } A_{\theta}\left(t-\tau_{\theta}\right) / A_{0} \\
\Rightarrow & T_{\text {steady }}^{\theta^{\max } \mid A_{\theta}}(\omega)=p_{\theta} \theta_{0}^{\max } e^{-j \omega \tau_{\theta}} / A_{0}
\end{aligned}
$$

where $T_{\text {steady }}^{\theta^{\max } \mid A_{\theta}}(\omega)=\hat{\theta}_{1}^{\max }(\omega) / \hat{A}_{\theta_{1}}(\omega)$ represents the transfer function between $\hat{\theta}_{1}^{\max }(\omega)$ and $\hat{A}_{\theta_{1}}(\omega)$.

\subsection{Transfer function $T_{\theta^{\max }}(\omega)$}

From the definitions of $\gamma_{\theta_{0}}$ and $\tau_{\theta}, \gamma_{\theta_{0}}$ can be rewritten as $\gamma_{\theta_{0}}=\tau_{\theta} \Gamma_{\theta}$ with $\Gamma_{\theta}=-(d \theta / d A) \Omega$. $\left(A_{0} / \theta^{\max }\left(A_{0}\right)\right)$, a dimensionless parameter characterizing the steady response of the quantity $\theta$ to strain rate. Then, by combining Eqs. (6) and (8), the transfer function $T_{\theta^{\max }}(\omega)$ between $\hat{\theta}_{1}^{\max }(\omega)$ and $\hat{a}_{1}(\omega)$ is given by $T_{\theta^{\max }}(\omega)=T_{\text {steady }}^{\theta^{\max } \mid A_{\theta}}(\omega) T_{\text {unst }}^{\text {finite, } \theta}(\omega)$. Gain and phase lag of $\theta^{\max }$ are expressed respectively by $G_{\theta^{\max }}(\omega)=$ $20 \log _{10}\left(\left|T_{\theta^{\max }}(\omega)\right| /\left|T_{\theta^{\max }}(\omega=0)\right|\right)$ and $\varphi_{\theta^{\max }}(\omega)$, where:

$$
\left\{\begin{aligned}
\left|T_{\theta^{\max }}(\omega)\right| & =\frac{\left|a_{1} p_{\theta} \theta_{0}^{\max }\right|}{A_{0}} \frac{1}{\sqrt{1+\eta(\omega)^{2}}} \frac{1}{\sqrt{1+\left(\frac{2 \eta(\omega) \Gamma_{\theta}}{D a_{\theta}}\right)^{2}}} \\
\varphi_{\theta^{\max }}(\omega) & =\tan ^{-1}(\eta(\omega))+\tan ^{-1}\left(2 \eta(\omega) \Gamma_{\theta} / D a_{\theta}\right) \\
& +2 \eta(\omega) / D a_{\theta}
\end{aligned}\right.
$$

with $\eta(\omega)=\omega /\left(2 A_{0}\right)=\pi f / A_{0}$ the Stokes number and $D a_{\theta}=A_{0}^{-1} \tau_{\theta}^{-1}$ the Damköhler number associated with $\theta$. From Eq. (9), it can be deduced that three nondimensional parameters are responsible for the phase lag and damping of the response of $\theta$ :

- $\eta$ compares the characteristic time associated to the strain rate $A_{0}$ to the imposed frequency $f$ and is responsible for the filtering of the flow structure. The damping response of all the quantities increases when $\eta$ increases.

- $D a_{\theta}$ is directly responsible for the phase lag and damping response due to the low chemical time scale of the analyzed quantity $\theta$. The lower $D a_{\theta}$ is, the more the response of $\theta^{\max }$ is phase-lagged and damped.

- $\Gamma_{\theta}$, which represents the steady response of the quantity $\theta$ to strain rate, also contributes to the damping response of $\theta$ with unsteady strain fluctuations. For high values of $\Gamma_{\theta}$, the damping response will be high.

This identified behavior is valid for all the quantities but is more significant in the case of species with large chemical characteristic time scales $\left(D a_{\theta} \ll 1\right)$, which is the case of soot precursors and particles. Equation (9) allows the predictions of the unsteady response of $\theta$ from information on steady flames. Its validity will discussed, in particular for soot production, in Sec. 4.3.

\section{Detailed modelisation of soot production}

Parallely to the asymptotic analysis, the behavior of pulsed laminar diffusion flames is investigated numeri- 
cally. In order to obtain an accurate numerical prediction of soot and its precursors, detailed models for both gas and solid phase are considered in this work and described below.

\subsection{Sectional method for solid phase}

The soot population is evaluated by using a sectional method. Each section $i$ represents particles with a volume between $V_{i}^{\min }$ and $V_{i}^{\max }$. The soot mass fraction $Y_{s, i}$ of the $i^{t h}$ section is given by the following transport equation:

$\frac{\partial \rho Y_{s, i}}{\partial t}+\nabla \cdot\left(\rho\left(\mathbf{u}+\mathbf{v}_{\mathbf{T}}\right) Y_{s, i}\right)=\nabla \cdot\left(\rho D_{s, i} \nabla\left(Y_{s, i}\right)\right)+\rho_{s} \dot{Q}_{s, i}$

where $\rho$ is the gas phase density, $\mathbf{u}$ is the gas velocity, $\mathbf{v}_{\mathbf{T}}$ is the thermophoretic velocity of the particles given in [18], $D_{s, i}$ is the diffusion coefficient of particles of the $i^{\text {th }}$ section defined in [19], $\rho_{s}$ is the constant soot density (chosen equal to $\rho_{s}=1860 \mathrm{~kg} / \mathrm{m}^{3}$ ) and $\dot{Q}_{s, i}$ is the production rate of the soot volume fraction for the $i^{\text {th }}$ section accounting for

nucleation, condensation, surface growth, oxidation and coagulation.

The models used to close this production rates are based on those used by Karkar el al. [20]. Several improvements have been made in the present work and are presented below.

Nucleation corresponds to the formation of the smallest solid particles. Here, the coalescence of two dimers is considered for the formation of these particles [21]. Condensation is considered as the coalescence of a dimer at a soot particle surface.

Dimers are formed from the collision of two polycyclic aromatic hydrocarbons (PAH). According to [22], only the collision of PAH with four-aromatic rings and more is considered. Here, seven PAH have been considered, from the pyrene $(A 4)$ and up to the coronen $(A 7)$. A quasi-steady-state hypothesis is considered between their production from the gaseous phase and their consumption by nucleation and condensation [20].

Soot surface reactions are responsible for both soot particles surface growth and oxidation. These phenomena are described through the HACA-RC mechanism [23]. The oxidation reaction by $\mathrm{OH}$ has been updated based on recent experimental results of [24].

According to previous works [21], big soot particles can not be considered as spherical particles. A soot particle of a given volume $V$ and surface $S$ is considered as a fractal aggregate of $n_{p}=S^{3} /\left(36 \pi V^{2}\right)$ primary spherical particles with a diameter $d_{p}=6 \mathrm{~V} / \mathrm{S}$. For each soot particle, $S$ is estimated by fitting numerical results from
[21] as a function of $V$ by $\left(S / S_{C_{2}}\right)=\left(V / V_{C_{2}}\right)^{2 / 3}$ for $V<V_{1}$ and $\left(S / S_{C_{2}}\right)=\left(V / V_{C_{2}}\right)^{\theta(V) / 3}$ for $V>V_{1}$, with $\theta(V)=2+0.175 \log _{10}\left(V / V_{1}\right)$ and $V_{1}=320 \mathrm{~nm}^{3}$. $V_{1}$ denotes the volume from which a soot particle is no longer considered as spherical. $S_{C_{2}}$ and $V_{C_{2}}$ are respectively the surface and volume of a spherical molecule composed of two carbon atoms.

Particle nucleation and condensation as well as coagulation are described through the Smoluchowski equation [25]. This equation is expressed as a function of the collision diameter $d_{c}$ of the soot particles, calculated as in [21] as a function of $d_{p}, n_{p}$ and their fractal dimension $D_{f}$ (chosen equal to $D_{f}=1.8$ ).

\subsection{Gaseous phase description, radiation model and solving strategy}

The detailed kinetic scheme KM2, due to [22], has been considered in this study. It involves 202 species and 1351 reactions, and has been validated for the estimation of PAH up to coronen.

A radiative source term has been added in the energy transport equation. It is computed at each point as a function of the absorbed and the emitted radiative powers [26], which are expressed as a function of the corresponding detailed intensities. Radiative properties are expressed via a narrow-band model [26]. For the gaseous species, $\mathrm{CO}_{2}, \mathrm{H}_{2} \mathrm{O}$ and $\mathrm{CO}$ are considered as the main contributors in radiation fluxes. For soot particles, the absorption coefficient $\kappa_{v \text {,soot }}=5.5 v f_{V}$ is estimated as a function of the soot volume fraction $f_{V}$ and the wave number $v$ [27].

The above models as well as Eqs.(10) are introduced into a system of 1-D equations describing a counterflow configuration based on self similar approximation and imposed variable strain rate or injection velocity [28]. The coupled gas and soot sections transport equations are solved using the REGATH package [29].

\subsection{Validation test cases}

The proposed modeling strategy has been first validated on soot prediction for:

- PSD description (not shown): numerical results show a fair agreement with the experimental data [30] obtained with the BSS (Burner Stabilized Stagnation) flame technique for laminar premixed ethylene flames.

- Response to steady strain rate (Fig. 1): the response of soot volume fraction as a function of the global steady strain rate has been reproduced numerically on the steady counterflow diffusion 
flame experimentally investigated by Decroix et al. [13] for three fuels.

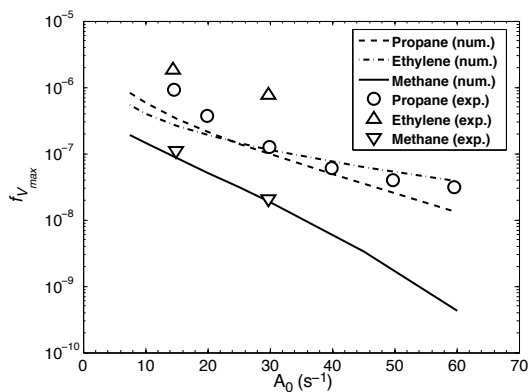

Figure 1. Maximum soot volume fraction $f_{V}^{\max }$ as a function of the global strain rate. Comparison between the present numerical (lines) and experimental (symbols) data from [13].

- Response to unsteady strain rate: an unsteady counterflow propane/air diffusion flame has been investigated by imposing an oscillating velocity at the injection as experimentally done in $[13,14]$. A phase lag of $125^{\circ}$ between the minimum average soot volume fraction and maximum imposed velocity was experimentally observed for $A_{0}=$ $15 \mathrm{~s}^{-1}, \alpha=30 \%$ and $f=\omega / 2 \pi=25 \mathrm{~Hz}$. With the numerical computation, a phase lag of $114^{\circ}$ was obtained.

The good agreement of the numerical results with experimental data confirms the validity of the retained modeling strategy.

\section{Detailed simulations of soot production in un- steady laminar diffusion flames at imposed strain rate}

\subsection{Numerical configuration}

A counterflow propane/air diffusion flame is considered here by varying the imposed strain rate $a(t)$ from an initial flame at $A_{0}=60 \mathrm{~s}^{-1}$. Ten frequencies and three amplitudes have been considered. Pure propane and pure air stream, both at $294 \mathrm{~K}$ are supplied through the two opposed nozzles at a distance $L=12.7 \mathrm{~mm}$, discretized with more than 400 points. For each studied frequency, ten signal periods were computed. Once the permanent regime was attained, the response of each variable was studied in terms of gain and phase lag.

\section{2. $P A H$ and soot particles response}

Results for small strain rate fluctuations ( $\alpha=10 \%)$ are considered here.

Figure 2 (left) presents the unsteady response of the soot maximum volume fraction and pyrene (A4) maximum mass fraction (the smallest considered soot precursor) to the unsteady imposed strain rate during two oscillating cycles. Quantities have been normalized with their respective steady values at the lowest and highest strain rates for three frequencies. The higher the frequency, the more $f_{V}^{\max }$ and $Y_{A 4}^{\max }$ fluctuations are dumped and phase-lagged. Looking at the results in the $a$-space (Fig. 2, right) enables a clear comparison with the quasi-steady solution (grey line). A quasi-steady response is observed at low frequency $(f=0.1 \mathrm{~Hz})$, while for higher frequencies, solutions step aside from the steady results.

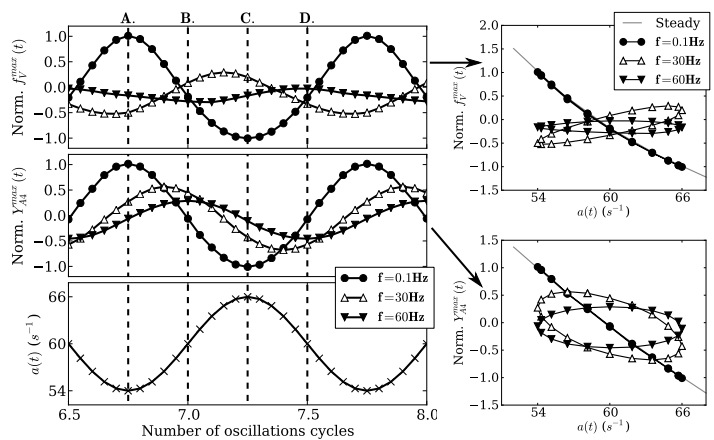

Figure 2. Normalized response of soot maximum volume fraction $\left(f_{V}^{\max }\right)$, pyrene maximum mass fraction $\left(Y_{A 4}^{\max }\right)$ to the unsteady imposed strain rate $(a(t))$.

The temporal evolution of the PSD is also studied here by looking at the four instants A.,B.,C.,D. of Fig. 2 separated by $90^{\circ}$ in one pulsation period. Results for three frequencies are presented in Fig. 2 together with the quasi-steady state at the spatial position $x^{\text {soot }}$, where soot volume fraction is maximum, close to the stagnation point. At each time, the PSD shows a bi-modal nature with one peak for small particles (generated by nucleation) and another for large aggregates (due to condensation and coagulation). In the quasi-steady case, from point $\mathbf{A}$. to $\mathbf{C}$. the characteristic flow time decreases (since $a(t)$ increases), so that particles have less time to coagulate. The position of the aggregates peak translates then towards smaller diameter values: the higher the strain rate, the smaller are the aggregates composing the soot population. Inversely, from point $\mathbf{C}$. to A., the strain rate decreases, particles have the time to coagulate and bigger aggregates populate the PSD. 
The unsteady PSDs follow such dynamics, but their response is affected by the phase-lag already observed on the global $f_{V}$. Indeed, at $f=5 \mathrm{~Hz}$, the PSD responds in a quasi-steady way, whereas the phase-lag effect is more and more evident on PSD for higher frequencies. The response of the PSD is also more and more damped so that at $f=60 \mathrm{~Hz}$ only small PSD fluctuations are observed between the four instants. For high oscillation frequencies, the PSD is observed to not oscillate anymore since the oscillations are completely damped.

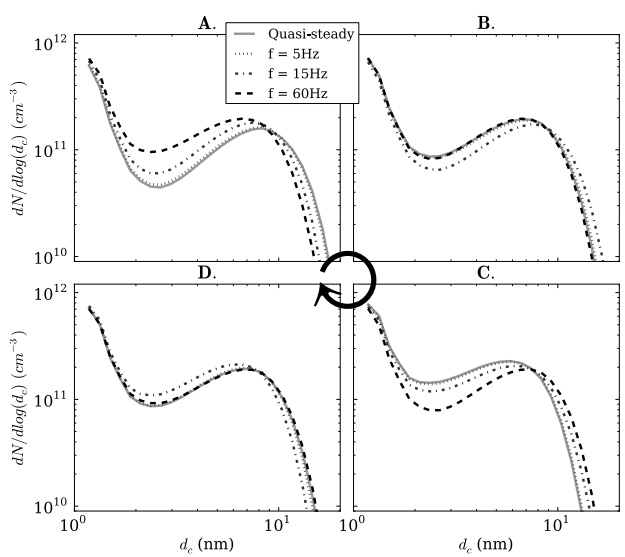

Figure 3. Unsteady variations of the PSD at $f_{V}^{\max }$ position.

\subsection{Comparison with analytical predictions}

In order to understand the processes governing the PSD evolution, results for the different sections are now investigated.

Figure 4 presents the response in terms of gain and phase lag of maximum temperature, $Y_{A 2}^{\max }, Y_{A 4}^{\max }$, maximum soot mass fraction of two sections (sections 12 and 16, whose mean diameter are indicated in Table 1) and $f_{V}^{\max }$. The response of precursors and soot is more phase-lagged and damped than temperature. Moreover, phase-lag and damping increases with their size (not shown for all precursors). Big particles are the main contributions to soot volume fraction, so that $f_{V}$ response is mainly governed by the last soot sections.

A good agreement is obtained between the numerical results (lines) and the analytical model (symbols) described in Section 2.2. Discrepancies are mainly observed at high frequencies but the hierarchical behavior between temperature, soot precursors and soot sections is well predicted. This confirms that soot dynamics are mainly governed by the three parameters identified with the analytical model. In particular, soot response is mainly due to its slow chemistry compared to the flame.
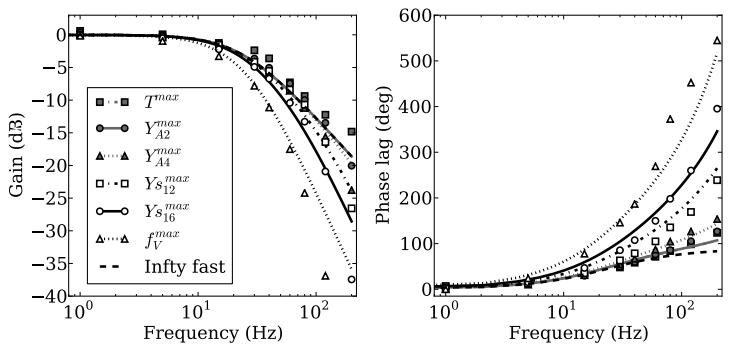

Figure 4. Comparison between analytical model predictions (lines) and numerical results (symbols) of amplitude gain and phase lag for maximum temperature, naphtalene $(A 2)$ and pyrene $(A 4)$ maximum mass fractions, maximum mass fractions of the $12^{\text {th }}$, the $16^{\text {th }}$ soot sections and maximum soot volume fraction. Analytical results for $T^{\max }$ and $Y_{A 2}^{\max }$ are superposed.

To identify the main physical processes contributing to such a long chemical time, the characteristic time scales for nucleation $\left(\tau_{\text {nu }}\right)$, condensation $\left(\tau_{\text {cond }}\right)$, surface growth $\left(\tau_{\mathrm{sg}}\right)$ and coagulation $\left(\tau_{\text {coag }}\right)$ have been estimated for different sections from the steady flame at $A_{0}$ following Appendix A. Table 1 presents these characteristic time scales normalized by the flame time scale $\left(\tau_{T}=0.31 \mathrm{~ms}\right)$ for five soot sections. All the characteristic time scales increase with the soot particle size, in particular for $\tau_{\text {cond }}$ and $\tau_{\text {coag }}$ which depend on the collisions rate. The particle number density of the last sections being smaller than for small particles sections, the number of particles available for collision is lower so that the characteristic time scales of collisional phenomena increases with the particle size.

The long characteristic time scale of $f_{V}^{\max }$, governing the phase-lag and damping of the unsteady response, is then mainly due to condensation and coagulation phenomena of the biggest particles.

\begin{tabular}{c|c||c|c|c|c}
\hline Section & $d_{c, i}^{\text {mean }}(\mathrm{nm})$ & $\frac{\tau_{\mathrm{nu}}}{\tau_{T}}$ & $\frac{\tau_{\text {cond }}}{\tau_{T}}$ & $\frac{\tau_{\mathrm{sg}}}{\tau_{T}}$ & $\frac{\tau_{\mathrm{coag}}}{\tau_{T}}$ \\
\hline \hline 1 & 1.2 & 3.4 & 3.2 & 3.6 & - \\
8 & 2.6 & - & 4.2 & 4.4 & 2.3 \\
12 & 4.2 & - & 6.5 & 5.7 & 4.6 \\
16 & 6.6 & - & 12 & 7.7 & 8.1 \\
20 & 10 & - & 17 & 8.3 & 13 \\
\hline
\end{tabular}

Table 1. Comparison of normalized characteristic time scales of nucleation, condensation, surface growth and coagulation. $d_{c, i}^{\text {mean }}$ represents the mean collisional diameter of a soot particle in the $i^{t h}$ section.

In order to study the validity of the assumption on the induced delay time due to slow chemistry presented in Section 2.2, Fig. 5 presents the obtained numerical phase lag due to this delay time as a function of the ex- 
pected one. This phase lag is obtained by substracting to the obtained numerical phase $\operatorname{lag} \varphi_{\theta^{\max }}^{\text {num }}(\omega)$ the theoretical phase lag of the equivalent steady strain rate $A_{\theta}$ of the variable of interest $\theta$. Good results are obtained for all the species and temperature, which confirms the representativity of the chosen variable $\left(\tau_{\theta}\right)$. Nevertheless, some discrepancies exist and future investigations are still needed in order to define in a more precise way this delay time.

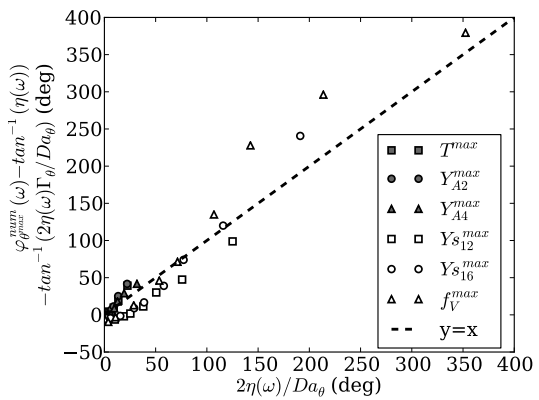

Figure 5. Numerical results of the induced phase lag due to the delay time imposed by slow chemistry as a function of the expected ones.

\subsection{Numerical results at high amplitudes}

In order to study the soot dynamics at higher amplitudes, computations have been performed for amplitudes $\alpha$ of $30 \%$ and $60 \%$. Table 2 compares the numerical results for the phase lag and amplitude gain of $Y_{A 4}^{\max }$ and $f_{V}^{\max }$ for three amplitudes and three frequencies. The phase lag increases with the frequency in a similar way for all the amplitudes. The gain remains almost the same for $\alpha=10 \%$ and $\alpha=30 \%$, but decreases for $\alpha=60 \%$. The difference of the numerical behavior between $\alpha=30 \%$ and $\alpha=60 \%$ highlights the non-linear effects for such amplitudes, which cannot be described by the linear analytical model whose predictions do not depend on the perturbation amplitude.

\section{Conclusions}

Response of sooting propane-air counterflow diffusion flames to imposed strain rate harmonic oscillations were numerically investigated with a detailed description for the gas and the solid phases. The unsteady behavior of soot particles and precursors production, as well as the PSD evolution, were studied both analytically and numerically. It has been observed that the higher the oscillation frequency is, the more PAHs and soot particles fluctuations are damped and phase-lagged so that unsteady solutions are farther and farther away

\begin{tabular}{|c|c|c|c|c|c|c|c|}
\hline \multirow{2}{*}{$\begin{array}{c}f \\
(\mathrm{~Hz})\end{array}$} & \multirow[t]{2}{*}{$\theta$} & \multicolumn{2}{|c|}{$\alpha=10 \%$} & \multicolumn{2}{|c|}{$\alpha=30 \%$} & \multicolumn{2}{|c|}{$\alpha=60 \%$} \\
\hline & & $G_{\theta_{\text {max }}}$ & $\varphi_{\theta^{\max }}$ & $G_{\theta^{\max }}$ & $\varphi_{\theta_{\max }}$ & $G_{\theta^{\max }}$ & $\varphi_{\theta^{\max }}$ \\
\hline 30 & $A 4$ & -4 & 54 & -4 & 53 & -5 & 56 \\
\hline 30 & $f_{V}$ & -4 & 146 & -4 & 158 & -13 & 142 \\
\hline 60 & $\overline{A 4}$ & -9 & 87 & -8 & 91 & -10 & 87 \\
\hline 60 & $f_{V}$ & -14 & 269 & -14 & 287 & -24 & 256 \\
\hline 120 & $A 4$ & -16 & 126 & -16 & 134 & -18 & 130 \\
\hline 120 & $f_{V}$ & -33 & 460 & -33 & 524 & -41 & 497 \\
\hline
\end{tabular}

Table 2. Numerical analysis of the impact of the strain fluctuation amplitude $(\alpha)$ on the pyrene maximum mass fraction and soot maximum volume fraction gain $(G$ in $d B)$ and phase lag ( $\varphi$ in $d e g$ ).

from the quasi-steady state. The phase-lag and damping increase with the size of PAHs and soot particles.

An analytical model has been proposed to predict the observed phase lags and dampings assuming a linear behavior. Three non-dimensional parameters $\left(\eta, D a_{\theta}\right.$ and $\left.\Gamma_{\theta}\right)$ govern the unsteady response. Soot particles are characterized by long time scales mainly due to condensation and coagulation phenomena. Indeed, compared to the gas species, their dynamics, particularly the additional identified phase lag, are mainly governed by the $D a_{\theta}$ parameter.

Therefore, models developped for numerical simulations of soot production in turbulent flames have to correctly reproduce these observed features in order to represent unsteady behaviors such as soot intermittency. On the one hand, these behaviors highlight the limits of flamelet regime assumption based on quasi-steady hypothesis, implying major complexities in modeling for turbulent calculations. In this sense, the presented results support the need for specific techniques [31] to account for PAHs response to unsteady strain rate fluctuations. On the other hand, the reduced models have to provide a good prediction of $\eta, D a_{\theta}$ and $\Gamma_{\theta}$ for PAHs and soot. As an example, representative soot precursors have to be chosen in terms of these three parameters in order to obtain the good unsteady behavior of soot production: large precursors dynamics (such as pyrene and coronen) have to be reproduced. The proposed analytical model will be very useful for the development of models that reproduce the dynamics of soot and their precursors in turbulent flames.

\section{AppendixA. Chemical characteristic time scales}

Species and flame characteristic time scales.

To study the unsteady response of each chemical species separately, the characteristic time scale $\tau_{k}$ for 
the $k^{\text {th }}$ species is defined as [32]:

$$
\tau_{k}=\left[X_{k}\right]^{\max } / \dot{\omega}_{k}^{\max }=\left(\rho Y_{k}\right)^{\max } /\left(W_{k} \dot{\omega}_{k}^{\max }\right)
$$

where $\left[X_{k}\right], Y_{k}, W_{k}$ and $\dot{\omega}_{k}$ are the molar concentration, the mass fraction, the molecular weight and the molar production rate of the $k^{\text {th }}$ species.

In the same way, the characteristic time scale $\tau_{T}$ of the flame can be defined as $\tau_{T}=\left(\rho c_{p} T\right)^{\max } / \dot{\omega}_{T}^{\max }$, with $c_{p}$ the mixture mass specific heat capacity and $\dot{\omega}_{T}$ the heat release rate.

\section{Soot sections characteristic time scales.}

As for the species, a time scale $\tau_{s, i}$ for the soot particles in the $i^{\text {th }}$ section can be defined as:

$$
\tau_{s, i}=\left(\rho Y_{s, i}\right)^{\max } /\left(\rho_{s}\left(\dot{Q}_{s, i}\right)^{\max }\right)
$$

By perturbing each volume fraction production rate relative to each phenomenon (ph) by a small value (typically $1 \%$ ), the characteristic time scales $\tau_{\mathrm{nu}, i}$ for nucleation, $\tau_{\text {cond }, i}$ for condensation, $\tau_{\mathrm{sg}, i}$ for surface growth, $\tau_{\mathrm{ox}, i}$ for oxidation and $\tau_{\mathrm{coag}, i}$ for coagulation can be expressed as:

$$
\tau_{\mathrm{ph}, i}=\Delta\left[\left(\rho Y_{s, i} / \rho_{s}\right)^{\max }\right] / \Delta\left[\left(\dot{Q}_{\mathrm{ph}, i}\right)^{\max }\right]
$$

where $\Delta\left[\left(\dot{Q}_{\mathrm{ph}, i}\right)^{\max }\right]$ corresponds to the variation of the peak volume fraction production rate of the phenomenon for the $i^{t h}$ section.

\section{References}

[1] J. Quaas, Nature 471 (7339) (2011) 456-457.

[2] M. D. Smooke, M. B. Long, B. C. Connelly, M. B. Colket, R. J. Hall, Combust. Flame 143 (4) (2005) 613-628.

[3] P. Desgroux, X. Mercier, K. A. Thomson, Proc. Combust. Inst. 34 (1) (2013) 1713-1738.

[4] V. Raman, R. O. Fox, Annual Review of Fluid Mechanics (2015) 159-190.

[5] A. Attili, F. Bisetti, M. E. Mueller, H. Pitsch, Combust. Flame 161 (7) (2014) 1849-1865.

[6] Y. Xin, J. P. Gore, Proc. Combust. Inst. 30 (1) (2005) 719-726.

[7] B. Franzelli, P. Scouflaire, S. Candel, Proc. Combust. Inst. 35 (2) (2015) 1921-1929.

[8] N. Peters, Prog. Energy Combust. Sci. 10 (3) (1984) 319-339.

[9] D. Veynante, L. Vervisch, Prog. Energy Combust. Sci. 28 (3) (2002) 193-266.

[10] B. Cuenot, F. Egolfopoulos, T. Poinsot, Combust. Theor. Model. 4 (1) (2000) 77-97.

[11] D. C. Haworth, M. C. Drake, S. B. Pope, R. J. Blint, Symposium (International) on Combustion 22 (1) (1989) 589-597.

[12] S. Candel, Proc. Combust. Inst. 29 (1) (2002) 1-28.

[13] M. E. Decroix, W. L. Roberts, Combust. Sci. Technol. 160 (1) (2000) 165-189.

[14] D. Santoianni, M. DeCroix, W. Roberts, Flow Turbul. Combust. 66 (1) (2001) 23-36.
[15] J. Xiao, E. Austin, W. L. Roberts, Combust. Sci. Technol. 177 (4) (2005) 691-713.

[16] A. Cuoci, A. Frassoldati, T. Faravelli, E. Ranzi, Combust. Flame 156 (10) (2009) 2010-2022.

[17] V. Huijnen, A. V. Evlampiev, L. M. T. Somers, R. S. G. Baert, L. P. H. de Goey, Combust. Sci. Technol. 182 (2) (2010) 103123.

[18] B. V. Derjaguin, A. I. Storozhilova, Y. I. Rabinovich, J. Colloid Interface Sci. 21 (1) (1966) 35-58.

[19] P. S. Epstein, Phys. Rev. 23 (1924) 710-733.

[20] D. Aubagnac-Karkar, J.-B. Michel, O. Colin, P. E. VervischKljakic, N. Darabiha, Combust. Flame 162 (8) (2015) 30813099.

[21] M. Mueller, G. Blanquart, H. Pitsch, Combust. Flame 156 (6) (2009) 1143 - 1155.

[22] Y. Wang, A. Raj, S. H. Chung, Combust. Flame 160 (9) (2013) 1667 - 1676.

[23] F. Mauss, T. Schäfer, H. Bockhorn, Combust. Flame 99 (3-4) (1994) 697 - 705, 25th Symposium (International) on Combustion Papers.

[24] F. Xu, A. M. El-Leathy, C. H. Kim, G. M. Faeth, Combust. Flame 132 (1-2) (2003) 43-57.

[25] M. Smoluchowski, Versuch einer mathematischen Theorie der Koagulationskinetik kolloider Lösungen, 1916.

[26] A. Soufiani, J. Taine, Int. J. Heat Mass Transfer 40 (4) (1997) 987-991.

[27] L. Tessé, F. Dupoirieux, J. Taine, Int. J. Heat Mass Transfer 47 (3) (2004) 555-572.

[28] N. Darabiha, N., Combust. Sci. Technol. 86 (1-6) (1992) 163181.

[29] B. Franzelli, B. Fiorina, N. Darabiha, Proc. Combust. Inst. 34 (1) (2013) 1659-1666.

[30] C. Saggese, S. Ferrario, J. Camacho, A. Cuoci, A. Frassoldati, E. Ranzi, H. Wang, T. Faravelli, Combust. Flame 162 (9) (2015) 3356-3369.

[31] Y. Xuan, G. Blanquart, Proc. Combust. Inst. 35 (2) (2015) 19111919.

[32] H. G. IM, J. H. Chen, J.-Y. Chen, Combust. Flame 118 (1-2) (1999) 204-212. 